Supporting Information for MS:

\title{
Thioarsenates in geothermal waters of Yellowstone National Park - determination, preservation, and geochemical importance
}

Britta Planer-Friedrich, Jacqueline London, R. Blaine McCleskey, D. Kirk Nordstrom, Dirk

Wallschläger

Number of pages: $\quad 10$

Number of figures: 0

Number of tables: $\quad 5$ 


\section{Electronic Annex EA1}

Sample site codes, locations, coordinates, and descriptions

\begin{tabular}{|c|c|c|c|c|}
\hline Code & Location & Name / Description & Latitude* & Longitude* \\
\hline GG01 & \multirow{2}{*}{$\begin{array}{l}\text { Gibbon Geyser Basin, } \\
\text { Gibbon Hill Group }\end{array}$} & unnamed surging hot spring & 4949602 & 0521509 \\
\hline GG02 & & unnamed hot spring & 4949509 & 0521502 \\
\hline GG03 & $\begin{array}{l}\text { Gibbon Geyser Basin, } \\
\text { Geyser Creek Group }\end{array}$ & "Bull’s Eye", surging hot spring & 4948596 & 0521456 \\
\hline GG04 & \multirow{4}{*}{$\begin{array}{l}\text { Gibbon Geyser Basin, } \\
\text { Sylvan Springs Group }\end{array}$} & unnamed hot spring & 4949724 & 0518583 \\
\hline GG05 & & unnamed hot spring & 4949644 & 0518589 \\
\hline GG06 & & unnamed hot spring & 4949524 & 0518341 \\
\hline GG07 & & Evening Primerose, hot spring & 4949593 & 0518365 \\
\hline GG08 & \multirow{2}{*}{$\begin{array}{l}\text { Gibbon Geyser Basin, } \\
\text { Geyser Creek Group }\end{array}$} & unnamed surging hot spring & 4948228 & 0521524 \\
\hline GG09 & & unnamed hot spring & 4948272 & 0521592 \\
\hline HL01 & Hazle Lake, north shore & $\begin{array}{c}\text { geothermal vents in shallow } \\
\text { lake }\end{array}$ & 4955315 & 0522788 \\
\hline HL02 & Hazle Lake, south shore & geothermal vent in shallow lake & 4955218 & 0522807 \\
\hline LG01 & \multirow{2}{*}{$\begin{array}{c}\text { Lower Geyser Basin, } \\
\text { Pocket Basin }\end{array}$} & Cavern Spring, hot spring & 4934167 & 0513178 \\
\hline LG02 & & Bath Spring, hot spring & 4934131 & 0513190 \\
\hline LG03 & \multirow{7}{*}{$\begin{array}{l}\text { Lower Geyser Basin, } \\
\text { right riverside }\end{array}$} & $\begin{array}{c}\text { Ojo Caliente, hot spring at } \\
\text { source (northeastern pool end) }\end{array}$ & \multirow{6}{*}{4934420} & \multirow{6}{*}{0512802} \\
\hline LG04 & & $\begin{array}{c}\text { Ojo Caliente, } 10.9 \text { m from } \\
\text { source (southwestern pool end) }\end{array}$ & & \\
\hline LG05 & & $\begin{array}{l}\text { Ojo Caliente, drainage, } 16.4 \mathrm{~m} \\
\text { from source }\end{array}$ & & \\
\hline LG06 & & $\begin{array}{l}\text { Ojo Caliente, drainage, } 22.4 \mathrm{~m} \\
\text { from source }\end{array}$ & & \\
\hline LG07 & & $\begin{array}{l}\text { Ojo Caliente, drainage, } 28.6 \mathrm{~m} \\
\text { from source }\end{array}$ & & \\
\hline LG08 & & $\begin{array}{l}\text { Ojo Caliente, drainage, } 35.4 \mathrm{~m} \\
\text { from source }\end{array}$ & & \\
\hline LG09 & & unnamed hot spring & 4933714 & 0513400 \\
\hline LG10 & $\begin{array}{l}\text { Lower Geyser Basin, } \\
\text { Pocket Basin }\end{array}$ & Azure Spring, hot spring & 4934193 & 0513262 \\
\hline LG11 & \multirow{2}{*}{$\begin{array}{c}\text { Lower Geyser Basin, } \\
\text { left riverside }\end{array}$} & unnamed geyser & 4934022 & 0513213 \\
\hline LG12 & & unnamed geyser & 4933953 & 0512389 \\
\hline LG13 & \multirow{6}{*}{$\begin{array}{l}\text { Lower Geyser Basin, } \\
\text { right riverside }\end{array}$} & $\begin{array}{c}\text { unnamed geyser at riverside, at } \\
\text { source }\end{array}$ & \multirow{6}{*}{4933698} & \multirow{6}{*}{0513326} \\
\hline LG14 & & $\begin{array}{l}\text { unnamed geyser at riverside, } \\
\text { drainage, } 3 \mathrm{~m} \text { from source }\end{array}$ & & \\
\hline LG15 & & $\begin{array}{l}\text { unnamed geyser at riverside, } \\
\text { drainage, } 5 \mathrm{~m} \text { from source }\end{array}$ & & \\
\hline LG16 & & $\begin{array}{l}\text { unnamed geyser at riverside, } \\
\text { drainage, } 7.5 \mathrm{~m} \text { from source }\end{array}$ & & \\
\hline LG17 & & $\begin{array}{l}\text { unnamed geyser at riverside, } \\
\text { drainage, } 10.5 \mathrm{~m} \text { from source }\end{array}$ & & \\
\hline LG18 & & $\begin{array}{l}\text { unnamed geyser at riverside, } \\
\text { drainage, } 15 \mathrm{~m} \text { from source }\end{array}$ & & \\
\hline
\end{tabular}




\begin{tabular}{|c|c|c|c|c|}
\hline Code & Location & Name / Description & Latitude & Longitude \\
\hline LG19 & \multirow{3}{*}{$\begin{array}{l}\text { Lower Geyser Basin, } \\
\text { Sentinel Meadows }\end{array}$} & Mound Spring, hot spring & 4934623 & 0511117 \\
\hline LG20 & & Steep Cone, hot spring & 4934825 & 0510858 \\
\hline LG21 & & Flat Cone, geyser & 4935029 & 0510839 \\
\hline NL01 & \multirow{4}{*}{ Nymph Lake } & $\begin{array}{l}\text { Frying Pan Spring, hot spring, } \\
\text { east of road }\end{array}$ & 4955502 & 0512992 \\
\hline NL02 & & $\begin{array}{l}\text { Frying Pan Spring, hot spring, } \\
\text { west of road }\end{array}$ & 4955446 & 0521965 \\
\hline NL03 & & $\begin{array}{l}\text { Roadside Spring West, hot } \\
\text { spring }\end{array}$ & 4955608 & 0521748 \\
\hline NL04 & & $\begin{array}{l}\text { Roadside Spring East, hot } \\
\text { spring }\end{array}$ & 4955613 & 0521777 \\
\hline RH01 & \multirow{5}{*}{$\begin{array}{l}\text { Norris Geyser Basin, } \\
\text { Ragged Hills }\end{array}$} & Crystal Spring, hot spring & 4952847 & 0522807 \\
\hline RH02 & & $\begin{array}{l}\text { Orpiment Puddle, sulfur-rich } \\
\text { small pool }\end{array}$ & 4952875 & 0522777 \\
\hline RH03 & & Lifeboat, surging hot spring & 4952734 & 0522615 \\
\hline RH04 & & Titanic, hot spring & 4952750 & 0522636 \\
\hline RH05 & & Persnickety, geyser & 4952746 & 0522657 \\
\hline RH06 & $\begin{array}{c}\text { Norris Geyser Basin, } \\
\text { Back Basin }\end{array}$ & Recess Spring, hot spring & 4952206 & 0522955 \\
\hline RH07 & \multirow{8}{*}{$\begin{array}{l}\text { Norris Geyser Basin, } \\
\text { Ragged Hills }\end{array}$} & "Milky Way II", hot spring & 4952681 & 0522578 \\
\hline RH08 & & $\begin{array}{l}\text { Cinder Pool } 1 \mathrm{~m} \text { depth, hot } \\
\text { spring }\end{array}$ & \multirow{2}{*}{4953276} & \multirow{2}{*}{0522971} \\
\hline RH09 & & $\begin{array}{c}\text { Cinder Pool } 14 \text { m depth, hot } \\
\text { spring }\end{array}$ & & \\
\hline RH10 & & Elk, hot spring & 4952948 & 0522812 \\
\hline RH11 & & Milky Way, hot spring & 4952799 & 0522702 \\
\hline RH12 & & $\begin{array}{l}\text { "Dragon Spring", hot spring at } \\
\text { source in yellow mats }\end{array}$ & \multirow{3}{*}{4953165} & \multirow{3}{*}{0522853} \\
\hline RH13 & & $\begin{array}{l}\text { "Dragon Spring" drainage } 7 \mathrm{~m} \\
\text { from source, in red mats }\end{array}$ & & \\
\hline RH14 & & $\begin{array}{l}\text { "Dragon Spring" drainage } 10 \mathrm{~m} \\
\text { from source in green mats }\end{array}$ & & \\
\hline
\end{tabular}

* coordinates are UTM, zone 12, date NAD 83 


\section{Electronic Annex EA2}

Arsenic speciation determined by AEC-ICP-MS; all concentrations in $\mu \mathrm{g} / \mathrm{L}$

\begin{tabular}{|c|c|c|c|c|c|c|c|c|c|c|}
\hline نٌ & 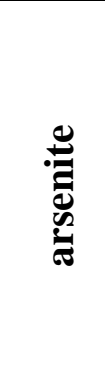 & 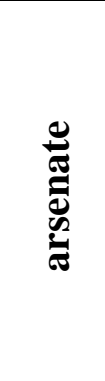 & 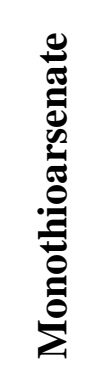 & 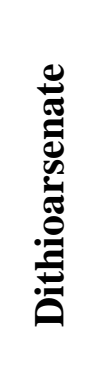 & 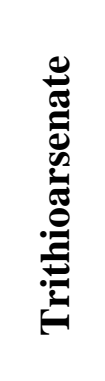 & 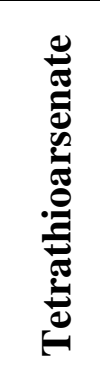 & 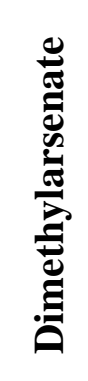 & 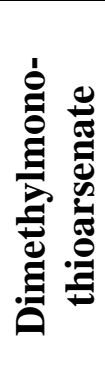 & 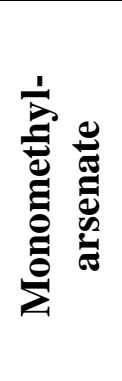 & 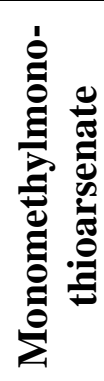 \\
\hline GG01 & 1030 & 23.6 & 17.3 & 115 & 510 & 1.26 & & & & \\
\hline GG02 & 2080 & 73.5 & 55.8 & 145 & 380 & & & & & \\
\hline GG03 & 2670 & 127 & 120 & 119 & 181 & & & & & \\
\hline GG04 & 800 & 45.2 & 28.9 & 168 & 412 & & & & & \\
\hline GG05 & 1980 & 10.3 & 1.80 & 8.38 & 1.80 & & & & & \\
\hline GG06 & 2920 & 149 & 46.5 & 53.8 & 8.02 & & & & & \\
\hline GG07 & 23.3 & 29.6 & 2.66 & 0.44 & & & 0.32 & & 1.62 & 0.15 \\
\hline GG08 & 142 & 261 & 201 & 430 & 1270 & 27.2 & & & & \\
\hline GG09 & 1590 & 35.9 & 68.8 & 183 & 326 & & & & & \\
\hline HL01 & 77.1 & 10.5 & 1.64 & 3.45 & & & 29.7 & 0.31 & 1.70 & 0.60 \\
\hline HL02 & 55.3 & 79.6 & 4.96 & 0.21 & & & 31.1 & 11.1 & 1.88 & 0.80 \\
\hline LG01 & 187 & 1120 & 10.6 & & & & & & & \\
\hline LG02 & 64.4 & 444 & 167 & 326 & 596 & 13.6 & & & & \\
\hline LG03 & 504 & 29.5 & 39.3 & 151 & 761 & & & & & \\
\hline LG04 & 694 & 34.0 & 54.3 & 183 & 624 & & & & & \\
\hline LG05 & 871 & 38.8 & 74.2 & 174 & 453 & & & & & \\
\hline LG06 & 1090 & 109 & 115 & 142 & 198 & & & & & \\
\hline LG07 & 1060 & 314 & 115 & 98.3 & 85.6 & & & & & \\
\hline LG08 & 905 & 554 & 104 & 89.2 & 52.9 & & & & & \\
\hline LG09 & 608 & 945 & 231 & 160 & 178 & & & & & \\
\hline LG10 & 279 & 632 & 530 & 78.2 & 98.7 & & & & & \\
\hline LG11 & 242 & 201 & 186 & 553 & 747 & & & & & \\
\hline LG12 & 198 & 364 & 219 & 343 & 525 & & & & & \\
\hline LG13 & 178 & 220 & 158 & 261 & 1330 & 50.5 & & & & \\
\hline LG14 & 221 & 207 & 248 & 239 & 1200 & 12.1 & & & & \\
\hline LG15 & 150 & 256 & 368 & 286 & 1060 & 10.8 & & & & \\
\hline LG16 & 174 & 343 & 476 & 317 & 909 & & & & & \\
\hline LG17 & 135 & 316 & 590 & 282 & 852 & & & & & \\
\hline LG18 & 190 & 446 & 755 & 237 & 465 & & & & & \\
\hline LG19 & 94.5 & 456 & 79.9 & 174 & 371 & 4.94 & & & & \\
\hline LG20 & 564 & 85.5 & 74.5 & 201 & 298 & & & & & \\
\hline LG21 & 67.4 & 99.2 & 212 & 392 & 163 & & & & & \\
\hline NL01 & 2.26 & 24.4 & 0.55 & 0.26 & & & 0.58 & & 0.99 & \\
\hline NL02 & 85.2 & 61.9 & 3.92 & 4.16 & & & 52.4 & 13.4 & 2.42 & 1.05 \\
\hline NL03 & 2640 & 349 & 78.8 & 89.1 & 65.3 & 1.15 & & & & \\
\hline NL04 & 306 & 75.0 & 1.45 & 3.49 & & & & & & \\
\hline RH01 & 2580 & 26.9 & 1.80 & 8.59 & 1.80 & & & & & \\
\hline RH02 & 8550 & 505 & 1100 & & & & & & & \\
\hline
\end{tabular}


Electronic Annex EA2 (cont.'d)

\begin{tabular}{|c|c|c|c|c|c|c|c|c|c|c|}
\hline نْ & 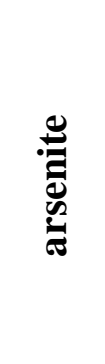 & 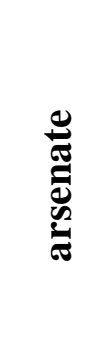 & 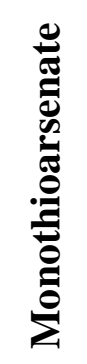 & 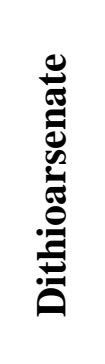 & 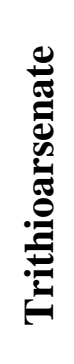 & 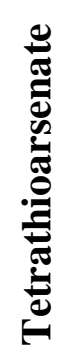 & 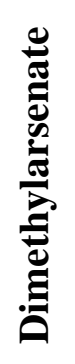 & 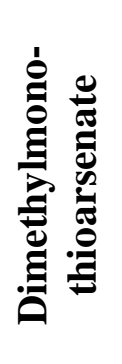 & 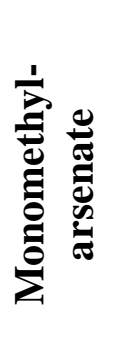 & 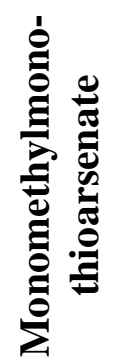 \\
\hline RH03 & 1380 & 3560 & 676 & & & & & & & \\
\hline RH04 & 510 & 5520 & 212 & & & & & & & \\
\hline RH05 & 5820 & 711 & 343 & & & & & & & \\
\hline RH06 & 2470 & 68.9 & 9.73 & 5.28 & & & & & & \\
\hline RH07 & 752 & 1360 & 29.4 & & & & & & & \\
\hline RH08 & 3020 & 16.4 & & 5.59 & & & & & & \\
\hline RH09 & 1740 & 102 & 26.0 & 8.13 & & & & & & \\
\hline RH10 & 2100 & 586 & 244 & & & & & & & \\
\hline RH11 & 767 & 157 & 474 & & & & & & & \\
\hline RH12 & 1100 & 84.0 & 73.2 & 8.77 & & & & & & \\
\hline RH13 & 299 & 896 & 8.44 & & & & & & & \\
\hline RH14 & 268 & 1210 & 17.2 & & & & & & & \\
\hline
\end{tabular}




\section{Electronic Annex EA3}

Selected basic water chemistry parameters for the sampled sites

\begin{tabular}{|c|c|c|c|c|c|c|c|c|c|c|c|c|c|c|}
\hline Cod & $\mathbf{H}$ & $\mathbf{m p}$ & pe & $\mathbf{O}_{2}$ & $\mathbf{S}^{2-}$ & ${ }_{2} \mathrm{O}_{3}$ & $\mathrm{Fe}^{2+}$ & $\mathrm{Fe}^{3+}$ & $\mathbf{N a}$ & $\mathbf{K}$ & $\mathbf{C a}$ & $\mathrm{Cl}^{-}$ & $\mathrm{O}_{4}{ }^{2-}$ & $\begin{array}{l}\text { Alka- } \\
\text { linity* }\end{array}$ \\
\hline & & & & & & 90 & .03 & 0.03 & 24 & 6.5 & 79 & 99 & 11 & 7 \\
\hline & & & & $J$ & & .02 & & $<0.03$ & 403 & 38.8 & 3.75 & 78 & 1.9 & 119 \\
\hline 200 & 7 & & & & & 13 & & $<0.03$ & 420 & 32.7 & 5.08 & 12 & & 3.3 \\
\hline & & & & & & 3.70 & & & 10 & 18.5 & .34 & 97 & ר? & 278 \\
\hline 05 & 0 & & & 7 & & 32.0 & & & 264 & 13.9 & 3.55 & 321 & 127 & 5.76 \\
\hline & 5 & & & 2 & & 2.35 & & & 453 & 51.0 & 3.75 & 567 & 165 & 77.8 \\
\hline & & & & & & 0.34 & & & 12.3 & 19.5 & 1.63 & 6.65 & 1040 & \\
\hline 08 & & & & 2 & & 1.79 & & & 473 & 20.3 & 0.38 & 407 & 136 & 339 \\
\hline & .4 & & & .4 & & 1.79 & .03 & & 353 & 20.7 & 3.97 & 18 & 104 & 15 \\
\hline & & & & & & & & & 12 & 36.1 & & & 107 & 9.9 \\
\hline & & & & & & 0.34 & & & 124 & 32.4 & 11.3 & 36 & 255 & \\
\hline LG 01 & & & & & & $<0.34$ & $<0.03$ & $<0.03$ & 323 & 10.6 & 0.77 & 05 & 3.1 & 159 \\
\hline & & & & & & & & & & & & & & 02 \\
\hline & & & & & & 0.34 & $<0.03$ & 0.03 & 350 & 8.78 & 0.81 & 27 & 9.3 & 200 \\
\hline LG 04 & & & 20 & & & 0.34 & $<0.03$ & $<0.03$ & 315 & 8.69 & 0.84 & 23 & 0.2 & 202 \\
\hline & & & & & & & & & & & & & & 94 \\
\hline & & & & & & & & & 326 & 8.77 & & 329 & 0.0 & 206 \\
\hline LG 07 & & & 1.9 & 1.8 & 23 & 0.34 & $<0.03$ & $<0.03$ & 331 & 8.79 & 0.81 & 337 & 0.4 & 209 \\
\hline & & & & & & & & & 364 & 11.7 & 0.88 & & .8 & 12 \\
\hline & & & & & & & & & 339 & & & 24 & 8.4 & 229 \\
\hline 10 & & & 2 & .2 & 1 & 48 & 03 & 1 & 308 & 11.6 & 1.13 & 14 & 6.5 & 174 \\
\hline & & & & & & & & & 39 & 13.9 & & 10 & 3.5 & 89 \\
\hline & & & & & & & & & 18 & 8.37 & & & 7.2 & 218 \\
\hline LG 13 & & & & & & .34 & .03 & & 359 & 14.4 & 0.48 & 311 & 9.6 & 239 \\
\hline & & & & & & & & & 355 & 13.6 & 0.50 & & 8.5 & 240 \\
\hline & & & & & & & & & 329 & 10.1 & 0.49 & & 9.7 & 242 \\
\hline & & & & & & & & & 362 & & & & 8.8 & 44 \\
\hline & & & & & & $<0.34$ & & & 337 & 11.5 & 0.52 & 311 & 9.4 & 244 \\
\hline & & & & & & & & & 331 & 11.7 & 0.53 & 18 & 2.3 & 241 \\
\hline & & & & & & & & & 325 & 11.2 & 0.32 & 236 & 14.3 & 251 \\
\hline & 3.1 & & -1.4 & & 0.64 & $<0.34$ & $<0.03$ & $<0.03$ & 295 & 9.81 & 0.21 & 254 & 13.3 & 248 \\
\hline & 8.5 & & -2.5 & & & $<0.34$ & & & 276 & 8.33 & 0.43 & 218 & 13.1 & 267 \\
\hline & & & & & & & & & 7.67 & 4.20 & 3.50 & 1.67 & 63.9 & \\
\hline NL 02 & 2.5 & 72 & 4.9 & 1.5 & 0.73 & 1.57 & 1.17 & $<0.03$ & 43.2 & 9.43 & 2.32 & 8.37 & 395 & \\
\hline & .7 & & 1.8 & 0.9 & & 7.62 & $<0.03$ & $<0.03$ & 418 & 8.04 & 1.42 & 425 & 66.8 & 157 \\
\hline NL 04 & .1 & 71 & 1.9 & 1.7 & 0.22 & $<0.34$ & 0.46 & $<0.03$ & 143 & 46.5 & 5.14 & 149 & 205 & \\
\hline
\end{tabular}


Electronic Annex EA3 (cont.'d)

\begin{tabular}{|l|c|c|c|c|c|c|c|c|c|c|c|c|c|c|}
\hline Code & $\mathbf{p H}$ & temp & $\mathbf{p e}$ & $\mathbf{O}_{\mathbf{2}}$ & $\mathbf{S}^{\mathbf{2 -}}$ & $\mathbf{S}_{\mathbf{2}} \mathbf{O}_{\mathbf{3}}$ & $\mathbf{F e}^{\mathbf{2 +}}$ & $\mathbf{F e}^{\mathbf{3 +}}$ & $\mathbf{N a}$ & $\mathbf{K}$ & $\mathbf{C a}$ & $\mathbf{C l}^{-}$ & $\mathbf{S O}_{\mathbf{4}}^{\mathbf{2 -}}$ & $\begin{array}{c}\text { Alka- } \\
\text { linity* }\end{array}$ \\
\hline RH 01 & 3.7 & 82 & 2.4 & 2.0 & 0.25 & 4.59 & 0.11 & 0.05 & 484 & 39.1 & 7.08 & 743 & 69.8 & \\
\hline RH 02 & 2.4 & 24 & 3.8 & 1.8 & 1.95 & $<0.34$ & 59.6 & $<0.03$ & 139 & 36.4 & 1.85 & 222 & 475 & \\
\hline RH 03 & 2.6 & 71 & 2.2 & 0.3 & 0.02 & $<0.34$ & 15.3 & 5.70 & 290 & 51.4 & 4.34 & 449 & 247 & \\
\hline RH 04 & 3.0 & 67 & 1.7 & 1.9 & 0.04 & $<0.34$ & 5.95 & 1.15 & 301 & 50.7 & 5.42 & 482 & 178 & \\
\hline RH 05 & 3.1 & 86 & 1.7 & 1.0 & 0.13 & $<0.34$ & 5.10 & 1.70 & 321 & 52.8 & 5.55 & 479 & 167 & \\
\hline RH 06 & 3.6 & 89 & 1.1 & 0.6 & 0.65 & 0.90 & 0.08 & 0.07 & 394 & 43.3 & 5.98 & 640 & 101 & \\
\hline RH 07 & 4.1 & 74 & 0.9 & 2.1 & 0.01 & $<0.34$ & 0.90 & 0.19 & 333 & 41.3 & 3.62 & 528 & 69.4 & \\
\hline RH 08 & 4.4 & 92 & 0.9 & 0.8 & 1.60 & 11.3 & 0.01 & 0.04 & 399 & 54.2 & 4.93 & 631 & 76.7 & \\
\hline RH 09 & 4.2 & 92 & 0.9 & 0.4 & 2.10 & 10.5 & $<0.03$ & 0.14 & 370 & 47.7 & 4.93 & 626 & 75.2 & \\
\hline RH 10 & 2.6 & 76 & 3.2 & 0.4 & $<0.01$ & $<0.34$ & 1.32 & 0.59 & 359 & 41.9 & 6.68 & 675 & 224 & \\
\hline RH 11 & 2.4 & 50 & 8.3 & 2.9 & 1.05 & $<0.34$ & 16.4 & 8.90 & 62.3 & 19.6 & 3.40 & 76.4 & 430 & \\
\hline RH 12 & 3.0 & 68 & 3.8 & 0.6 & 3.10 & $<0.34$ & 1.92 & 0.09 & 277 & 42.4 & 4.76 & 495 & 142 & \\
\hline RH 13 & 3.9 & 53 & 4.3 & 3.4 & $<0.01$ & $<0.34$ & 1.69 & 0.28 & 323 & 47.6 & 5.10 & 501 & 149 & \\
\hline RH 14 & 3.9 & 47 & 4.2 & 4.1 & $<0.01$ & $<0.34$ & 1.47 & 0.61 & 328 & 44.4 & 5.09 & 506 & 151 & \\
\hline
\end{tabular}

* concentrations are in $\mathrm{mg} / \mathrm{L}$; Alkalinity is in $\mathrm{mg} / \mathrm{L} \mathrm{CaCO}_{3}$; temp is the source temperature in ${ }^{\circ} \mathrm{C}$; as described in the text, samples were cooled down to temperatures of less than $50^{\circ} \mathrm{C}$ to increase sensor long-term performance; samples that were still above $50^{\circ} \mathrm{C}$ after passing the $50 \mathrm{~m}$ PTFE hose were not measured for oxygen because of the sensitivity of the optical sensor towards high temperatures; pe was calculated as $0.0169 *[\mathrm{EMF}+(-0.7443 *$ temp+224.98)] 


\section{Electronic Annex EA4}

Calculated S:As ratios for mono-, di-, tri- and tetrathioarsenate

\begin{tabular}{|c|c|c|c|c|c|c|c|c|c|c|c|c|}
\hline \multirow[b]{2}{*}{ Code } & \multicolumn{3}{|c|}{ Monothioarsenate } & \multicolumn{3}{|c|}{ Dithioarsenate } & \multicolumn{3}{|c|}{ Trithioarsenate } & \multicolumn{3}{|c|}{ Tetrathioarsenate } \\
\hline & $\begin{array}{c}\text { As } \\
{[\mu \mathrm{M}]}\end{array}$ & $\underset{[\mu \mathrm{M}]}{\mathrm{S}}$ & S:As & $\begin{array}{c}\text { As } \\
{[\mu \mathrm{M}]}\end{array}$ & $\left.\begin{array}{c}\mathbf{S} \\
{[\mu \mathrm{M}]}\end{array}\right]$ & S:As & $\begin{array}{c}\text { As } \\
{[\mu \mathrm{M}]}\end{array}$ & $\underset{[\mu \mathrm{M}]}{\mathbf{S}}$ & S:As & $\begin{array}{c}\text { As } \\
{[\mu \mathrm{M}]}\end{array}$ & $\underset{[\mu \mathrm{M}]}{\mathrm{S}}$ & S:As \\
\hline MEAN & & & 0.95 & & & 1.92 & & & 2.99 & & & 25.6 \\
\hline STD & & & 0.10 & & & \begin{tabular}{|l|l|}
0.14 \\
\end{tabular} & & & 0.23 & & & \begin{tabular}{|l|}
10.9 \\
\end{tabular} \\
\hline RSD \% & & & 11 & & & 7 & & & 8 & & & 43 \\
\hline No. & & & 17 & & & 21 & & & 26 & & & 5 \\
\hline & & & & & & & & & & & & \\
\hline GG01 & 0.23 & & & 1.53 & & & 6.81 & 19.2 & 2.82 & 0.02 & & \\
\hline GG02 & 0.74 & & & 1.93 & & & 5.07 & 15.3 & 3.01 & & & \\
\hline GG03 & 1.59 & & & 1.59 & & & 2.41 & 7.00 & 2.90 & & & \\
\hline GG04 & 0.39 & & & 2.24 & & & 5.49 & 16.5 & 3.00 & & & \\
\hline GG05 & 0.02 & & & 0.11 & & & 0.02 & & & & & \\
\hline GG06 & 0.62 & & & 0.72 & & & 0.11 & & & & & \\
\hline GG07 & 0.04 & & & 0.01 & & & & & & & & \\
\hline GG08 & 2.69 & & & 5.73 & 11.8 & 2.06 & 16.9 & 49.3 & 2.91 & 0.36 & 5.80 & \begin{tabular}{|l|l|}
16.1 \\
\end{tabular} \\
\hline GG09 & 0.92 & & & 2.44 & & & 4.35 & 9.21 & 2.12 & & & \\
\hline HL01 & 0.02 & & & 0.05 & & & & & & & & \\
\hline HL02 & 0.07 & & & & & & & & & & & \\
\hline LG01 & 0.14 & & & & & & & & & & & \\
\hline LG02 & 2.23 & 4.08 & $1.83 *$ & 4.35 & 8.18 & \begin{tabular}{|l|l}
1.88 \\
\end{tabular} & 7.95 & 24.5 & 3.08 & 0.18 & 3.60 & \begin{tabular}{|l|}
19.9 \\
\end{tabular} \\
\hline LG03 & 0.52 & & & 2.01 & 3.33 & 1.65 & 10.2 & 31.9 & 3.14 & & & \\
\hline LG04 & 0.72 & & & 2.44 & 4.78 & 1.96 & 8.32 & 26.9 & 3.23 & & & \\
\hline LG05 & 0.99 & 1.09 & 1.10 & 2.33 & 3.88 & 1.67 & 6.04 & 18.8 & 3.12 & & & \\
\hline LG06 & 1.54 & 1.47 & 0.95 & 1.90 & 3.59 & 1.89 & 2.63 & \begin{tabular}{|l|}
7.73 \\
\end{tabular} & 2.94 & & & \\
\hline LG07 & 1.53 & 2.84 & $1.85^{*}$ & 1.31 & 2.55 & 1.94 & 1.14 & 3.66 & 3.21 & & & \\
\hline LG08 & 1.39 & 1.18 & 0.85 & 1.19 & 2.59 & 2.18 & 0.71 & 2.00 & 2.84 & & & \\
\hline LG09 & 3.08 & 2.71 & 0.88 & 2.13 & 4.17 & 1.96 & 2.38 & 6.13 & 2.58 & & & \\
\hline LG10 & 7.06 & 6.81 & 0.96 & 1.04 & 2.17 & 2.08 & 1.32 & 4.16 & 3.16 & & & \\
\hline LG11 & 2.48 & 1.99 & 0.80 & 7.38 & \begin{tabular}{|l|}
14.2 \\
\end{tabular} & 1.92 & 9.96 & 30.7 & 3.09 & & & \\
\hline LG12 & 2.92 & 2.26 & 0.77 & 4.57 & 8.84 & 1.93 & 7.00 & \begin{tabular}{|l|}
20.7 \\
\end{tabular} & 2.95 & & & \\
\hline LG13 & 2.11 & 2.23 & 1.06 & 3.48 & 6.86 & \begin{tabular}{|l|l|}
1.97 \\
\end{tabular} & 17.8 & 54.0 & 3.04 & 0.67 & 14.2 & 21.1 \\
\hline LG14 & 3.31 & 2.80 & 0.85 & 3.18 & 6.32 & 1.99 & 16.0 & 51.1 & 3.20 & 0.16 & 4.4 & \begin{tabular}{|l|}
27.1 \\
\end{tabular} \\
\hline LG15 & 4.91 & 4.82 & \begin{tabular}{|l|}
0.98 \\
\end{tabular} & 3.82 & 6.66 & \begin{tabular}{|l}
1.74 \\
\end{tabular} & 14.1 & 44.2 & 3.13 & 0.14 & 6.3 & \begin{tabular}{|l|l|}
43.8 \\
\end{tabular} \\
\hline LG16 & 6.35 & 6.05 & 0.95 & 4.23 & 7.69 & 1.82 & 12.1 & 37.3 & 3.07 & & & \\
\hline LG17 & 7.87 & 8.49 & 1.08 & 3.76 & 7.91 & 2.10 & 11.4 & 35.5 & 3.12 & & & \\
\hline LG18 & 10.1 & 9.89 & 0.98 & 3.16 & 6.14 & 1.94 & 6.21 & 18.8 & 3.03 & & & \\
\hline LG19 & 1.06 & & & 2.32 & \begin{tabular}{|l|l|}
4.07 \\
\end{tabular} & 1.75 & 4.95 & 14.7 & 2.98 & 0.07 & & \\
\hline LG20 & 0.99 & & & 2.68 & 5.36 & 2.00 & 3.98 & 11.8 & 2.97 & & & \\
\hline LG21 & 2.83 & 2.97 & 1.05 & 5.23 & 10.1 & 1.93 & 2.17 & 6.67 & 3.08 & & & \\
\hline NL01 & 0.01 & & & & & & & & & & & \\
\hline NL02 & 0.05 & & & 0.06 & & & & & & & & \\
\hline NL03 & 1.05 & & & 1.19 & & & 0.87 & & & 0.02 & & \\
\hline NL04 & 0.02 & & & 0.05 & & & & & & & & \\
\hline RH01 & 0.02 & & & 0.11 & & & 0.02 & & & & & \\
\hline
\end{tabular}


Electronic Annex EA4 (cont.'d)

\begin{tabular}{|c|c|c|c|c|c|c|c|c|c|c|c|c|}
\hline \multirow[b]{2}{*}{ Code } & \multicolumn{3}{|c|}{ Monothioarsenate } & \multicolumn{3}{|c|}{ Dithioarsenate } & \multicolumn{3}{|c|}{ Trithioarsenate } & \multicolumn{3}{|c|}{ Tetrathioarsenate } \\
\hline & $\begin{array}{c}\text { As } \\
{[\mu \mathrm{M}]}\end{array}$ & $\begin{array}{c}\mathbf{S} \\
{[\mu \mathrm{M}]}\end{array}$ & S:As & $\begin{array}{c}\mathbf{A s} \\
{[\mu \mathrm{M}]}\end{array}$ & $\begin{array}{c}\mathbf{S} \\
{[\mu \mathrm{M}]}\end{array}$ & S:As & $\begin{array}{c}\text { As } \\
{[\mu \mathrm{M}]}\end{array}$ & $\begin{array}{c}\mathbf{S} \\
{[\mu \mathrm{M}]}\end{array}$ & S:As & $\begin{array}{c}\mathbf{A s} \\
{[\mu \mathbf{M}]}\end{array}$ & $\begin{array}{c}\mathbf{S} \\
{[\mu \mathrm{M}]}\end{array}$ & S:As \\
\hline RH02 & 14.7 & 12.7 & 0.87 & & & & & & & & & \\
\hline RH03 & 9.01 & 9.62 & 1.07 & & & & & & & & & \\
\hline RH04 & 2.83 & & & & & & & & & & & \\
\hline RH05 & 4.58 & & & & & & & & & & & \\
\hline RH06 & 0.13 & & & 0.07 & & & & & & & & \\
\hline RH07 & 0.39 & & & & & & & & & & & \\
\hline RH08 & & & & 0.07 & & & & & & & & \\
\hline RH09 & 0.35 & & & 0.11 & & & & & & & & \\
\hline RH10 & 3.25 & 3.05 & 0.94 & & & & & & & & & \\
\hline RH11 & 3.14 & & & 0.05 & & & & & & & & \\
\hline RH12 & 0.98 & & & 0.12 & & & & & & & & \\
\hline RH13 & 0.11 & & & & & & & & & & & \\
\hline RH14 & 0.23 & & & & & & & & & & & \\
\hline
\end{tabular}

* $\mathrm{SO}^{+}$trace of monothioarsenate peaks showed interference by another peak with similar retention time for the samples LG02 and LG07; that led to two high S:As ratios; these values were not considered for ratio calculations since they are obviously biased 


\section{Electronic Annex EA5}

Comparison between sum of arsenic species and total arsenic concentrations in unpreserved, $\mathrm{HNO}_{3}$ and

$\mathrm{HCl}$ preserved samples*

\begin{tabular}{|c|c|c|c|c|c|c|c|c|}
\hline & \multicolumn{4}{|c|}{ total arsenic concentration $[\mu \mathrm{g} / \mathrm{L}]$} & \multicolumn{4}{|c|}{ ratios [\%] } \\
\hline Code & $\begin{array}{c}\sum \text { As } \\
\text { species }\end{array}$ & $\begin{array}{c}\text { un- } \\
\text { preserved }\end{array}$ & $\begin{array}{c}\mathrm{HNO}_{3} \\
\text { preserved }\end{array}$ & $\begin{array}{c}\mathrm{HCl} \\
\text { preserved }\end{array}$ & $\begin{array}{c}\Sigma / \\
\text { unpres }\end{array}$ & $\begin{array}{l}\mathrm{HNO}_{3} / \\
\text { unpres. }\end{array}$ & $\begin{array}{c}\text { HCl / } \\
\text { unpres. }\end{array}$ & $\begin{array}{c}\text { HCl / } \\
\text { HNO3 }\end{array}$ \\
\hline GG01 & 1,700 & 1,900 & 1,990 & 780 & 89 & 104 & 41 & 39 \\
\hline GG02 & 2,730 & 2,620 & 2,670 & 2,330 & 104 & 102 & 89 & 87 \\
\hline GG03 & 3,210 & 3,060 & 3,190 & 2,380 & 105 & 104 & 78 & 75 \\
\hline GG04 & 1,460 & 1,590 & 1,690 & 419 & 91 & 106 & 26 & 25 \\
\hline GG05 & 2,010 & 1,750 & 1,870 & 1,570 & 115 & 107 & 90 & 84 \\
\hline GG06 & 3,180 & 3,070 & 3,180 & 2,700 & 103 & 103 & 88 & 85 \\
\hline GG07 & 58.1 & 59.1 & 63.6 & 53.8 & 98 & 108 & 91 & 84 \\
\hline GG08 & 2,330 & 2,430 & 2,520 & 342 & 96 & 104 & 14 & 14 \\
\hline GG09 & 2,200 & 2,280 & 2,290 & 1,200 & 97 & 100 & 53 & 52 \\
\hline HL01 & 125 & 123 & 135 & 90.9 & 102 & 109 & 74 & 67 \\
\hline HL02 & 185 & 218 & 250 & 140 & 85 & 114 & 64 & 56 \\
\hline LG01 & 1,320 & 1,340 & 1,380 & 1,190 & 99 & 103 & 89 & 87 \\
\hline LG02 & 1,610 & 1,590 & 2,030 & 181 & 101 & 128 & 11 & 9 \\
\hline LG03 & 1,490 & 1,490 & 1,540 & 586 & 100 & 103 & 39 & 38 \\
\hline LG04 & 1,590 & 1,450 & 1,570 & 714 & 109 & 108 & 49 & 46 \\
\hline LG05 & 1,610 & 1,520 & 1,570 & 1,150 & 106 & 104 & 76 & 73 \\
\hline LG06 & 1,660 & 1,550 & 1,610 & 1,260 & 107 & 104 & 81 & 78 \\
\hline LG07 & 1,670 & 1,590 & 1,630 & 1,370 & 105 & 102 & 86 & 84 \\
\hline LG08 & 1,710 & 1,600 & 1,660 & 1,530 & 107 & 104 & 96 & 92 \\
\hline LG09 & 2,120 & 1,980 & 2,040 & 1,910 & 107 & 103 & 97 & 94 \\
\hline LG10 & 1,620 & 1,550 & 1,600 & 1,480 & 104 & 103 & 95 & 93 \\
\hline LG11 & 1,930 & 1,800 & 2,470 & 392 & 107 & 137 & 22 & 16 \\
\hline LG12 & 1,650 & 1,650 & 1,720 & 377 & 100 & 104 & 23 & 22 \\
\hline LG13 & 2,200 & 2,110 & 2,040 & 323 & 105 & 97 & 15 & 16 \\
\hline LG14 & 2,130 & 2,100 & 2,070 & 497 & 101 & 99 & 24 & 24 \\
\hline LG15 & 2,130 & 2,180 & 2,220 & 526 & 98 & 102 & 24 & 24 \\
\hline LG16 & 2,220 & 2,150 & 2,180 & 742 & 103 & 101 & 34 & 34 \\
\hline LG17 & 2,180 & 2,150 & 2,210 & 1,060 & 101 & 103 & 50 & 48 \\
\hline LG18 & 2,090 & 2,140 & 2,190 & 1,400 & 98 & 103 & 66 & 64 \\
\hline LG19 & 1,180 & 1,180 & 1,190 & 679 & 100 & 101 & 58 & 57 \\
\hline LG20 & 1,220 & 1,180 & 1,220 & 995 & 104 & 103 & 84 & 82 \\
\hline LG21 & 933 & 988 & 1,010 & 800 & 95 & 102 & 81 & 79 \\
\hline NL01 & 29.0 & 24.0 & 21.5 & 21.5 & 121 & 90 & 90 & 100 \\
\hline NL02 & 224 & 184 & 166 & 168 & 122 & 90 & 91 & 102 \\
\hline NL03 & 3,230 & 3,460 & 3,390 & 2,800 & 93 & 98 & 81 & 83 \\
\hline NL04 & 386 & 388 & 384 & 320 & 99 & 99 & 83 & 83 \\
\hline RH01 & 2,620 & 2,840 & 2,890 & 2,620 & 92 & 102 & 92 & 91 \\
\hline RH02 & 10,200 & 14,300 & 14,600 & 13,700 & 71 & 102 & 96 & 94 \\
\hline RH03 & 5,620 & 5,890 & 8,320 & 8,490 & 95 & 141 & 144 & 102 \\
\hline RH04 & 6,240 & 6,650 & 6,630 & 7,160 & 94 & 100 & 108 & 108 \\
\hline RH05 & 6,870 & 6,490 & 6,540 & 6,920 & 106 & 101 & 107 & 106 \\
\hline
\end{tabular}

Electronic Annex EA5 (cont.'d) 


\begin{tabular}{|c|c|c|c|c|c|c|c|c|}
\hline & \multicolumn{4}{|c|}{ total arsenic concentration $[\mu \mathrm{g} / \mathrm{L}]$} & \multicolumn{4}{|c|}{ ratios [\%] } \\
\hline Code & $\begin{array}{c}\sum \text { As } \\
\text { species }\end{array}$ & $\begin{array}{c}\text { un- } \\
\text { preserved }\end{array}$ & $\begin{array}{c}\mathrm{HNO}_{3} \\
\text { preserved }\end{array}$ & $\begin{array}{c}\text { HCl } \\
\text { preserved }\end{array}$ & $\begin{array}{c}\Sigma / \\
\text { unpres }\end{array}$ & $\begin{array}{l}\mathrm{HNO}_{3} / \\
\text { unpres. }\end{array}$ & $\begin{array}{c}\text { HCl / } \\
\text { unpres. }\end{array}$ & $\begin{array}{c}\text { HCl / } \\
\text { HNO3 }\end{array}$ \\
\hline RH06 & 2,560 & 2,180 & 2,210 & 1,230 & 117 & 101 & 56 & 56 \\
\hline RH07 & 2,140 & 2,050 & 1,980 & 1,970 & 105 & 97 & 96 & 99 \\
\hline RH08 & 3,040 & 2,540 & 2,680 & 1,120 & 120 & 105 & 44 & 42 \\
\hline RH09 & 1,880 & 2,290 & 3,820 & 308 & 82 & 167 & 13 & 8 \\
\hline RH10 & 2,930 & 3,030 & 3,150 & 2,880 & 97 & 104 & 95 & 91 \\
\hline RH11 & 1,400 & 1,690 & 1,480 & 994 & 83 & 88 & 59 & 67 \\
\hline RH12 & 1,270 & 1,170 & 1,310 & 413 & 109 & 112 & 35 & 32 \\
\hline RH13 & 1,200 & 1,220 & 1,490 & 1,480 & 99 & 122 & 122 & 99 \\
\hline RH14 & 1,490 & 1,530 & 1,550 & 1,470 & 98 & 101 & 96 & 95 \\
\hline
\end{tabular}

* concentrations of unpreserved samples were determined by ICP-MS in September (analysis of one sample consists of 3 replicates; 12 samples re-run 10 days later showed mean deviations from initial concentrations of 1.0\%; 20 samples re-run on ICP-AES showed mean deviations from ICP-MS results of $3.7 \%$ )

concentrations of $\mathrm{HNO}_{3}$ preserved samples are mean values from two ICP-MS determinations in July and September (analysis of one sample consists of 3 replicates; 24 samples re-run in July showed mean deviations from initial (July) concentrations of 1.2\%, 9 samples re-run in September showed mean deviations from initial (September) concentrations of $2.3 \%$; comparison between samples analyzed in July and September showed mean deviations of $4.1 \%$ for 42 samples, 8 samples (GG01, GG04, GG08, LG02, LG11, LG12, LG15, RH09) were significantly higher in September (for those samples mean values were replaced by September values only); in comparison to samples analyzed by ICP-AES the final ICP-MS concentrations showed mean deviations of 8.0\%)

concentrations of $\mathrm{HCl}$ preserved samples were determined by HG-AAS (23 samples re-run two days later showed mean deviations from initial concentrations of 4.7\%; 20 samples re-run on ICP-AES for comparison showed mean deviations from HG-AAS of $5.5 \%$ ) 